\title{
ESCUCHANDO EL SONIDO: CONCEPCIONES SOBRE ACÚSTICA EN ALUMNOS DE DISTINTOS NIVELES EDUCATIVOS
}

\author{
PERALES PALACIOS, F.J. \\ Departamento de Didáctica de Ias Ciencias Experimentales. Facultad de Ciencias de la Educación. \\ Universidad de Granada. Campus Universitario de Cartuja. 18071 Granada.
}

\begin{abstract}
SUMMARY
In recent years, many articles have been published on students' preconceptions of different theoretical concepts in Physics. Howewer, one area of this discipline which has received very little attention is that of Acoustics. This article first examines Acoustics from a wide range of perspectives, placing it within a scientific, didactic, and sociological framework. Secondly, it analyzes the results of a test given to students of different ages and levels in order to identify common preconceptions of Acoustics. Finally a reflection about the possible implications these might have for the Physics classroom is made.
\end{abstract}

\section{INTRODUCCIÓN}

Roig (1982) denominaba su trabajo como El sonido, un tema olvidado. Otros autores como Lanzarica y otros (1978), Juncos del Egido y Pérez de Landazábal (1985) o Linder (1993) corroboran ese titular con datos relativos al gran porcentaje de profesores que, ante la escasez de tiempo para impartir los programas de ciencias, optan por excluir los tópicos relativos al sonido.

Este hecho contrasta sin embargo con la incidencia social de los fenómenos acústicos. Hoy día son mayoritarios los alumnos que cuentan en su hogar con una minicadena, un walkman ...; conocen el manejo de algún instrumento musical; graban su propia voz; etc. Por otro Iado, la tecnología actual se sirve del sonido y sus fundamentos para lanzar al mercado instrumentos con las más diversas aplicaciones: generadores ultrasónicos para el tratamiento de las nubes tóxicas o para ahuyentar insectos o roedores, el oído electrónico para la mejora de la capacidad auditiva o el tratamiento de la dislexia infantil, las ecografías para la revisión fetal, el micros- copto acústico, etc. Tales evidencias confirman lamentablemente el abismo existente entre lo que la escuela tradicional ofrece y lo que la sociedad demanda del individuo (algo ya señalado por Lord, 1981).

Esta carencia no es exclusiva del ámbito escolar sino que también afecta a la investigación educativa. Parece exis tir un mimetismo perfecto entre ambos campos por cuanto se produce una alarmante escasez de trabajos que, dentro de la óptica constructivista, se ocupen de indagar en las nociones previas de los alumnos sobre acústica. De hecho y, como describiremos más adelante, sólo hemos localizado una decena de citas bibliográficas en esta línea mediante distintas consultas en la biliograf́a internacional. Esto supone a nuestro juicio un importante hueco en el inventario de las concepciones fúsicas que se viene llevando a cabo desde hace varios años por numerosos investigadores, de cara al diseño de estrategias de enseñanza-aprendizaje vinculadas con los conocimientos previos y los intereses de los individuos. De 
Tabla I

Porcentajes aproximados de presencia de conceptos acústicos en la muestra de libros analizada (entre paréntesis figuran denominaciones alternativas con una menor frecuencia de aparición).

\begin{tabular}{|l|c|}
\hline CONCEPTOS DE ACÚSTICA PRESENTES & PORCENTAJE DE APARICIÓN \\
\hline Sonido / intensidad del sonido (de las ondas sonoras) & $100 \%$ \\
\hline Ondas sonoras / velocidad del sonido (de las ondas sonoras) / timbre / tono & $80 \%$ \\
\hline Sonido débil (suave) & $65 \%$ \\
\hline $\begin{array}{l}\text { Compresión (ondas de) / enrarecimiento (ratificación) / sonido fuerte / } \\
\text { reverberación / sonido agudo / sonido grave / ultrasonidos (ondas ultra- } \\
\text { sónicas) / diapasón }\end{array}$ & $50 \%$ \\
\hline $\begin{array}{l}\text { Sensación sonora / sonido puro / resonancia / tiempo de reverberación / } \\
\text { umbral de audición (de sensación desagradable) / intensidad fisiologica } \\
\text { o subjetiva (auditiva) / intensidad física (objetiva) / cco / } \\
\text { micrófono / campo de audición (de audibilidad) / infrasonidos } \\
\text { (ondas infrasónicas) / reflexión del sonido (factor de reflexión) / } \\
\text { espectro acústico (espectrograma del sonido) / amplitud de la presión } \\
\text { (de los cambios de presion) }\end{array}$ & \\
\hline
\end{tabular}

aquí que nuestro objetivo sea contribuir a suplir parcialmente esa ausencia y romper el silencio educativo que acompaña al sonido.

El desarrollo de la investigación propuesta se ha enmarcado en la metodología utilizada en otros trabajos previos en el campo de la óptica geométrica (Perales, 1987; Perales y Nicvas, 1990). Es decir, partiendo de un análisis del contenido científico a tres bandas: estructural, didáctico y sociológico, se confeccionará un test coherente con dicho análisis, seguido de su aplicación y el análisis de los resultados.

\section{ANÁLISIS DE CONTENIDOS}

\section{Análisis estructural}

Este encabezamiento debe incluir, a nuestro parecer, una reflexión previa sobre el tópico científico a investigar que desemboque en una propuesta de estructura conceptual y secuencial de los contenidos objeto de atención. Esto permitiría al profesor o al investigador reflexionar sobre la identificación de las concepciones previas, la jerarquización de los contenidos en aras de su aprehensión por parte de los alumnos, la evaluación de los mismos, etc. No siempre resulta fácil la traslación de estructura lógica a estructura psicológica, fucnte de muchas tensiones en la enseñanza de las ciencias; pero estimamos imprescindible el estudio de la primera antes de proceder a la toma de decisiones.

Siguiendo un proceso similar al descrito en otro trabajo (Perales, 1987), vamos a proceder a seleccionar los conceptos acústicos más comunes (frecuencia de aparición $>35 \%$ ) en seis libros de física de educación secundaria y primer año de universidad (Anexo I), lo que se presenta por orden de frecuencia decreciente en la tabla $I$.

Un análisis más fino de dichos conceptos permite establecer dos tipos de relaciones intraconceptuales: la de atributo y la de subconcepto. Esta última da lugar a la representación en forma arborescente simbolizada en la figura 1, quedando la tabla II para la relación de atributo.

Las dos últimas representaciones contemplan todos los conceptos incluidos en la tabla $I$, a excepción de diversos instrumentos (diapasón y micrófono) y de algunos conceptos del ámbito físiológico (sensación sonora y campo de audición).

Tabla I1

Relaciones concepto-atributo establecidas entre los conceptos de la tabla I (* concepto extracústico).

\begin{tabular}{|l|l|}
\hline $\begin{array}{l}\text { CONCEPTO } \\
\text { PRINCIPAL }\end{array}$ & \multicolumn{1}{|c|}{ ATRIBUTO } \\
\hline Sonido (ondas sonoras) & $\begin{array}{l}\text { Intensidad / valocidad / timbre } \\
\text { / tono / espectro / reflexión / eco } \\
\text { / resonancia }\end{array}$ \\
\hline Reverberación & Tiempo \\
\hline Presion* & Amplitud \\
\hline
\end{tabular}


Figura I

Representación arborescente de las relaciones concepto-subconcepto presentes en los conceptos de la tabla I (compuesto: subconcepto no contemplado en la tabla I; presión y umbral: conceptos extracústicos)
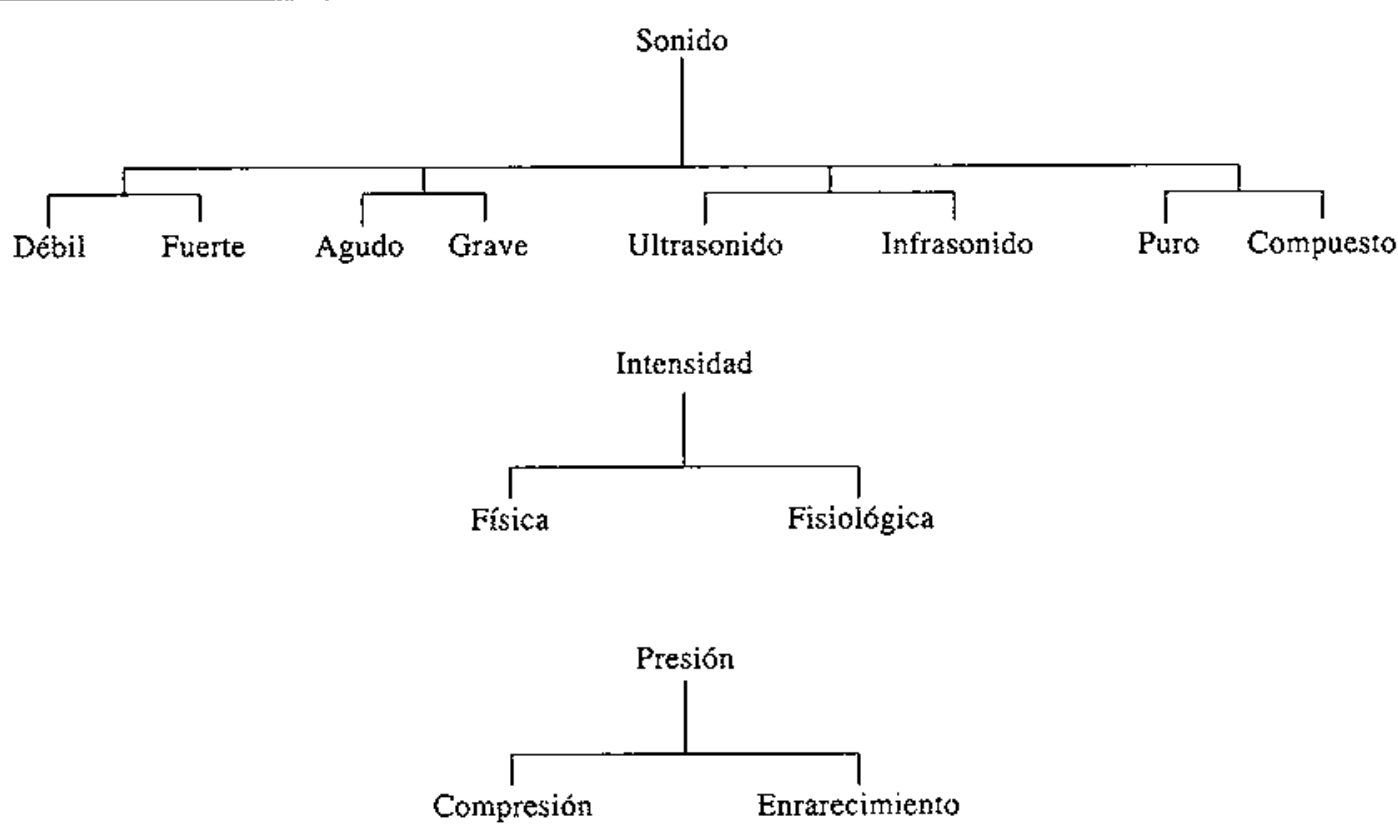

En cuanto a la secuencia de contenidos, tomaremos como referencia la organización temporal que se muestra en los índices de los ejemplares bibliográficos analizados. Para ello fue preciso agrupar tales índices en unidades con un cierto grado de coherencia (por ejemplo, emisión del sonido, propagación del sonido, etc.). Ante todo nos encontramos con una importante discrepancia en el modo de secuenciar los contenidos sonoros $y$, por otra parte, con una frecuente imbricación entre el estudio específico del sonido y el de las ondas en general. Prácticamente están presentes toda clase de combinaciones en la ordenación temporal de los contenidos, reiterándose únicamente las secuencias siguientes:

Propagación del sonido $\rightarrow$ Velocidad del sonido

Velocidad del sonido $\rightarrow$ Cualidades del sonido

Reflexión $\rightarrow$ Eco

En la figura 2 recogemos una propuesta de secuenciación de los princípales tópicos seleccionados en la revi- sión bibliográfica, para lo cual hemos tomado las siguientes premisas (que trataremos de ir justificando en los próximos apartados del trabajo):

- EI sonido debe quedar enmarcado en el contexto del movimiento ondulatorio que, junto al movimiento de partículas, constituye uno de los mecanismos básicos de propagación de la energía en Ia naturaleza.

- El sentido secuencial lógico debería corresponderse con el sensorial; esto es, el sonido se emite, se propaga y se detecta.

- EI sonido constituye una fuente de comunicación social de primera magnitud, así como un fenómeno con múltiples implicaciones tecnológicas.

\section{Análisis didáctico}

Este análisis pretende, en una primera fase, identificar y sistematizar los distintos puntos de vista con que la 
Eigura 2

Propuesta de secuenciación temporal para los contenidos de actística.

\section{Movimiento ondulatorio $\downarrow$}

Emisión del sonido $\downarrow$

Propagación del sonido. Ondas sonoras $\downarrow$

$$
\text { Ecuación }
$$

$\downarrow$

Magnitudes

(velocidad, frecuencia, energía, intensidad) $\downarrow$

Propiedades

(reflexión y eco, refracción, interferencias, difracción, efecto Doppler) $\downarrow$

Detección del sonido $\downarrow$

\section{El oído}

$\downarrow$

Cualjdades fisiológicas

$\downarrow$

Aplicaciones

$\downarrow$

Sonido musical (grabación, reproducción) $\downarrow$

Ondas estacionarias $\downarrow$

Acústica arquitectónica

ULtrasonidos $\downarrow$

Otras

(ecografias, cálculos renales, oídos electrónicos...)

literatura educativa ha abordado la acústica. Es decir, enfoques conceptuales, experiencias de demostración, etc.

En una segunda fase, tal análisis podría hacerse extensivo al tratamiento que este tópico recibe por parte de libros de texto de uso común en los distintos niveles educativos, incidiendo en las distintas variables inherentes: lenguaje utilizado, metodología de exposición, problemas sugeridos, etc.
Dadas las limitaciones de espacio de este trabajo, vamos a circunscribirnos a la primera fase. Para ello hemos procedido a consultar tres fuentes. La primera es Ia extensa monografía de Driver y Watts (1990) que revisa la prolija base de datos relativa a concepciones de los alumnos en linea con los presupuestos constructivistas. De todos los artículos reseñados, sólo nos fue posible hallar uno relativo al sonido (Linder y Erickson, 1989; recientemente el primer autor ha publicado sendos trabajos: Linder, 1992, 1993). La segunda consulta se centró en la base de datos ERIC entre los años 19831993, dando lugar a la identificación de un total de 19 referencias que, una vez analizadas, hemos agrupado en torno a los siguientes elementos:

Currículos que incluyen la acústica: 5 artículos.

Instrumentos generadores de sonido: 4 artículos.

Enseñanza/aprendizaje de algunos tópicos específicos: 4 artículos.

Sonido musical: 2 artículos.

Otros: 2 artículos.

Finalmente, la tercera consulta se efectuó a través de la revisión de Pfundt y Duit (1994, 4a. ed.), centrándose, al igual que en el caso de Driver y Watts (1990), sobre marcos alternativos de los estudiantes. Se detectaron diez trabajos distribuidos del modo siguiente:

Artículos de revista específicos de acústica: 4 .

Artículos de revista con otros tópicos: 2 .

Comunicaciones a congresos: 4 .

Se inciluye aquí el artículo encontrado en la obra de Driver y Watts (1990).

En cuanto a los trabajos prácticos sobre acústica, una revisión de nueve libros con esa temática (Anexo II) nos ha permitido identificar y agrupar las experiencias propuestas de acuerdo con la clasificación expuesta cn la tabla III.

\section{Análisis sociológico}

Este apartado pretende reflejar la conexión de los fenómenos sonoros con el entorno en que normalmente se desenvueiven los alumnos. Efectivamente el sonido, a pesar de la marginación que padece en el ámbito curricular y en la investigación educativa, posee un indudable efecto motivador derivado de su conexión con los intereses de los alumnos, En un primer avance podríamos identificar los siguientes núcleos de conexión social:

\section{Emisión del sonido}

- La voz

\section{Propagación del sonido}

- El eco 


\section{INVESTIGACIÓN DIDÁCTICA}

Tabla III

Agrupamiento de los trabajos prácticos incluidos en una muestra bibliográfica (Anexo II) de acueróo con el dominio conceptual implicado.

\begin{tabular}{|l|l|r|}
\hline FASE SONORA & DOMINIO CONCEPTUAL & FRECUENCIA DE APARFCIÓN \\
\hline \multirow{2}{*}{ EMISIÓN } & Instrumentos musicales & 11 \\
& Voz & 3 \\
\hline PROPAGACION & Otros & 19 \\
& En distintos medios & 12 \\
& Ondas sonoras & 5 \\
& Intensidad & 3 \\
& Velocidad & 3 \\
\hline DETECCION & Reflexión (reverberación y $\varepsilon c 0)$ & 1 \\
\hline & Difracción & 16 \\
& Oído & 6 \\
\hline
\end{tabular}

\section{Detección del sonido}

- Artificial: micrófono, osciloscopio

- Natural: el oído

- Identificación de sonidos

\section{Relaciones sonido-música}

- Reproducción musical: discos de vinilo, discos compactos, casetes, grabadoras y reproductoras de sonido - Instrumentos musicales: guitarra, piano, órgano, etc.

- Canto: tipos de voz

\section{Sonido-medioambiente}

- Contaminación acústica.
Creemos que en esta relación el profesor puede hallar una primera fuente de inspiración para promover la motivación hacia su estudio y relacionarlo con las experiencias previas de los alumnos para, al fin, lograr un ambiente positivo de trabajo derivado de la creencia en la utilidad de los contenidos que se aborden.

En definitiva, y aun reconociendo la primacia de la imagen como medio de transmisión de la información en sus distintas variantes (televisión, cine, publicidad, etc.), el sonido ocupa un lugar nada desdeñable en la vida cotidiana e implica unas mayores cotas de reflexión y participación (radio, conversación, etc.).

Tabla IV

Datos de las muestras de alumnos participantes en el estudio.

\begin{tabular}{|c|c|c|c|c|c|c|}
\hline \multirow[t]{2}{*}{ MUESTRA } & \multirow[t]{2}{*}{$N$} & \multicolumn{2}{|c|}{ EDAD } & \multicolumn{2}{|c|}{ SEXO } & NIVEL EDUCATIVO \\
\hline & & $\bar{x}$ & $\sigma_{n-1}$ & Mujeres & Hombres & \\
\hline M1 & 33 & 12,64 & 0,78 & $30,3 \%$ & $69,7 \%$ & $7^{\circ}$ de Ènseñanza Básica \\
\hline $\mathrm{M} 2$ & 33 & 15,45 & 0,57 & $60,7 \%$ & $39,3 \%$ & $2^{\circ}$ Educación Secundaria \\
\hline M3 & 26 & 17,61 & 0,85 & $34,6 \%$ & $65,4 \%$ & Pre-Universitarto \\
\hline M4 & 48 & 21,49 & 1,57 & $56.2 \%$ & $43,8 \%$ & $2^{\circ}$ de Magisterio \\
\hline
\end{tabular}




\section{DETECCIÓN DE IDEAS PREVIAS}

\section{Muestras de alumnos}

Los alumnos participantes totalizaron un número de 140 agrupados en cuatro muestras distintas cuyos datos más significativos se recogen en la tabla IV. Las cuatro muestras estaban ubicadas en distintos centros educativos de la provincia de Granada y su nivel social en todos los casos era medio-bajo.

\section{Test}

El problema de la construcción de un instrumento de diagnóstico que reproduzca fielmente el pensamiento del alumno está lejos de ser resuelto, en la medida en que cualquiera de ellos sufre un destacado grado de incerti- dumbre inherente a toda medida educativa; es más, la elección de uno u otro va a influir notablemente en los resuitados logrados. Es por ello que hemos optado por un test multiforme que combina ítems de distinta naturaleza y que, por tanto, debe servir para identificar distintas dimensiones del conocimiento, tanto conceptual como procesual.

Por un lado, el test debiera incluir cuestiones sobre los conceptos sonoros más relevantes y que, en los niveles educativos en que se mueve este trabajo, podrían ser los siguicntes:

- Sonido (naturaleza)

- Atributos sonoros: intensidad y tono/frecuencia

- Velocidad del sonido

- Ondas sonoras.

Figura 3

Red conceptual representativa del test. Los números representan los items de éste.

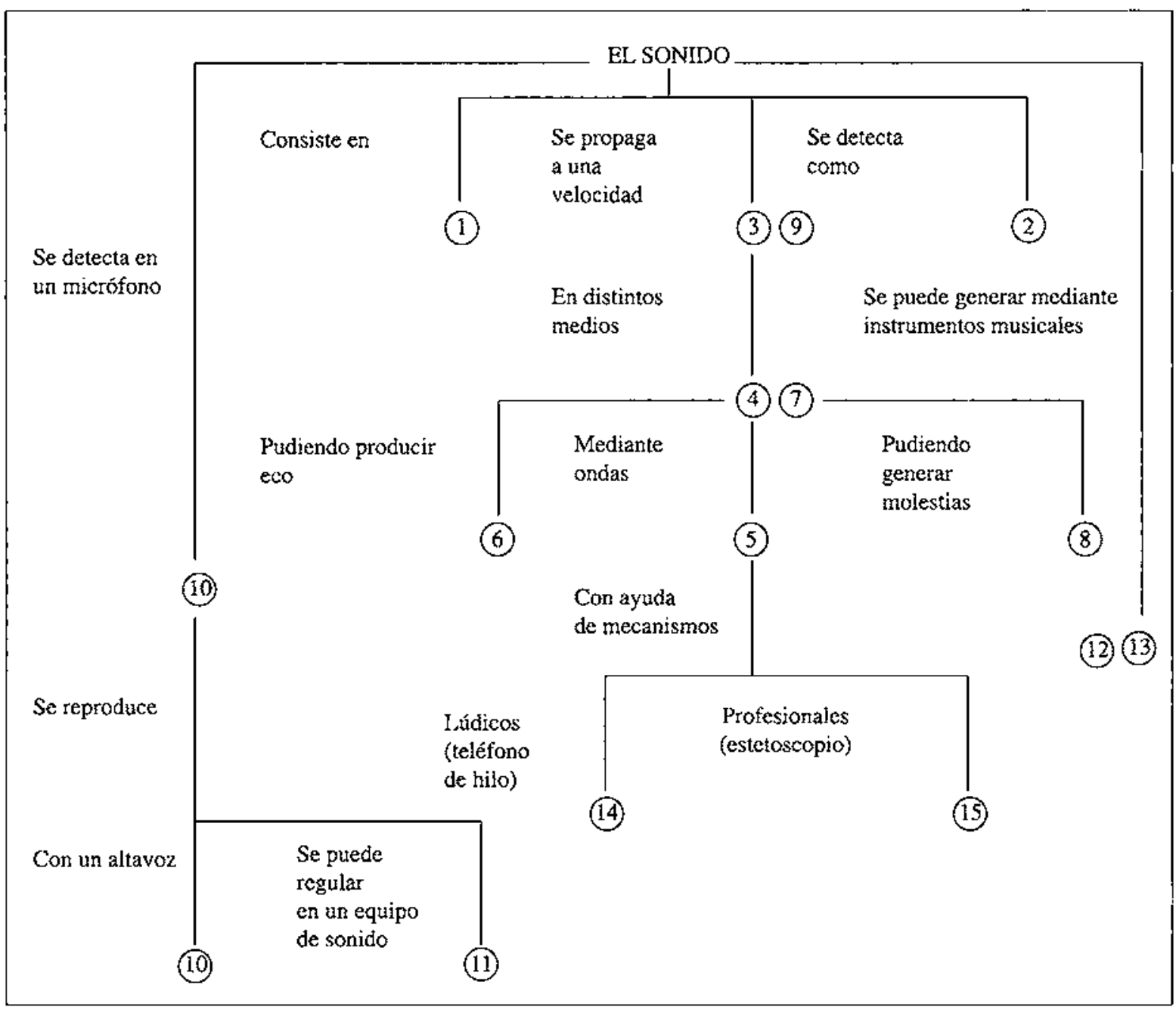


Esta relación podría ser complementada con otros conceptos de especial relevancia en la vida cotidiana del alumno:

- Eco
- Ruido/Contaminación acústica
- Trueno
- Micrófono
- Altavoz
- Instrumentos musicales.

Si atendemos al conocimiento procesual y fenomenológico, deberían incorporarse experiencias relativamente frecuentes en el ámbito del alumno. Hemos seleccionado algunas de cllas:

- Sonidos distintos producidos por un mismo cuerpo

- Sonidos producidos por distintos cuerpos

- Teléfono de hilo.

- Estetoscopio.

En cuanto a Ia naturaleza de los ítems, éstos constaban de cuestiones de respuesta abierta e ftems gráficos que debían ser interpretados (interview-about-instances, véase, por ejemplo, Stead y Osborne, 1980), aunque en nuestro caso por escrito.

Con estas premisas se confeccionó el test que aparece en el anexo III y cuya red conceptual representativa se muestra en la figura 3 .

\section{RESULTADOS}

El test fue pasado en el mismo año a todas las submuestras de alumnos por sus profesores habituales y de forma que no hubieran recibido enseñanza formal de acústica durante ese curso académico.

Los datos extraídos se han analizado de acuerdo con los siguientes criterios:

- Concepciones de los alumnos más significativas

- Evolución de las mismas en función del nivel educativo o edad de la muestra

En una primera etapa se han ido tabulando los resultados obtenidos en cada ítem del test para cada una de las muestras. Las tablas V y VI recogen, a título de ilustración, los resultados extraídos de sendos ítems.

\section{DISCUSIÓN DE LOS RESULTADOS}

Procederemos al análisis de los resultados agrupando los ítems de acuerdo con las siguientes categorías (Fig. 3):

- Naturaleza y descripción del sonido (ítems I y 2).

- Propagación del sonido (ítems $3,4,5,6,7$ y 9).
- Contaminación acústica (ítem 8).

- Tecnología y aplicaciones acústicas (ítems 10, 11, 12, 13,14 y 15 ).

Este análisis, lo centraremos en la naturaleza científica de las propias respuestas, su evolución a través de las distintas muestras y su posible casuística.

\section{Naturaleza y descripción del sonido}

De forma análoga a como ocurre con la luz, el sonido es identificado por su causa (emisión) y por sus efectos (detección), aunque también se hace como una entidad en el espacio (propagación), por lo que representa un avance con relación a lo que ocurre con la luz (Perales, 1994). En este sentido, la evolución por edad o nivel educativo es evidente: el sonido pasa de ser un "ruido» a una propagación de ondas en el espacio material. Esto supone en sí mismo un avance cualitativo importante, por cuanto existen ya indicios de atribuir la propagación al concurso de las partículas del medio, aunque la naturaleza de las ondas dista mucho de ser comprendida.

Tal evolución se manifiesta iguaimente en los resultados del ítem 2 en cuanto a la riqueza semántica del término sonido que, de ser considerado mayoritariamente como ruido por los alumnos más jóvenes, se convierte en un glosario de adjetivos relativos a sus cualidades en los de mayor edad. Asimismo es de destacar la significativa presencia de la «música» como descriptor del sonido también en los individuos de menor edad.

La educación musical recibida por los alumnos de la muestra M4 puede estar detrás de estos últimos resultados y la propia disciplina de la física -ambas cursadas durante el anterior año académico al que se les pasó este test-puede explicar sus referencias a los movimientos de las partículas justificativos del sonido.

\section{Propagación del sonido}

Ésta ha sido contemplada en el pretest desde diversas perspectivas: velocidad de propagación (item 3), propagación en medios materiales (ítems 5,7 y 9) y en el vacío (ítem 4) y, finalmente, Ja reflexión o eco (f́tem 6).

- Velocidad de propagación: sólo los alumnos de los niveles educativos superiores conocen de un modo mayoritario el valor aproximado de la velocidad del sonido en el aire (f́tem 3). En cuanto a su comparación con la velocidad de la luz (ítem 9), para la mayoría de los alumnos resulta evidente que la velocidad del sonido es menor que aquélla (en un porcentaje mayor que el descrito por Lucas, 1988), pero persisten dificultades en Jos dos primeros niveles, lo que se traduce en una apreciable ausencia de respuestas y en una presunta simultaneidad del fenómeno luminoso (ael sonido tarda un tiempo en recorrer el espacion), secuencialidad (cel rayo se ve primero y después liega el sonidon) o atribución sensorial ( «se ve antes porque la vista es más rápida que el oído"). 
Tabla $\mathrm{V}$

Respuestas dadas por los alumnos al ítem 5 relativo a la naturaleza de una onda expansiva (en el agrupamiento de las respuestas no se recogen aquéllas con porcentajes menores del I0 por ciento).

\begin{tabular}{|c|c|c|c|}
\hline $\begin{array}{l}\text { MUESTRA } \\
\text { (Porcentaje de respuestas) }\end{array}$ & $\begin{array}{l}\text { AGRUPAMIENTO } \\
\text { DE LA RESPUESTA }\end{array}$ & $\begin{array}{l}\text { PORCENTAJE } \\
\text { DE RESPUESTAS }\end{array}$ & $\begin{array}{l}\text { EJEMPLIFICACION IDE } \\
\text { LA RESPUESTA }\end{array}$ \\
\hline $\mathrm{Ml}(87,9 \%)$ & $\begin{array}{l}\text { Propagación por aire } \\
\text { Emisión } \\
\text { Propagación por tierra }\end{array}$ & $\begin{array}{l}37,9 \% \\
31 \% \\
20,7 \%\end{array}$ & $\begin{array}{l}\text { Las ondas producidas son muy fuertes y pueden } \\
\text { romper objetos de vidrio } \\
\text { Se debe al gran ruido de la explosion } \\
\text { Una onda que se produce con tanta fuerza que hace } \\
\text { retumbar las viviendas y las destruye }\end{array}$ \\
\hline M2 $(90,9 \%)$ & $\begin{array}{l}\text { Propagacion por aire } \\
\text { Emisión } \\
\text { Emisión + Detección }\end{array}$ & $\begin{array}{l}43,8 \% \\
25 \% \\
12,5 \%\end{array}$ & $\begin{array}{l}\text { Ondas que propagan el sonido } \\
\text { Ruido tan potente que rompió los cristales } \\
\text { Alcance del artefacto }\end{array}$ \\
\hline $\operatorname{M3}(92,3 \%)$ & $\begin{array}{l}\text { Propagación por aire } \\
\text { Emisión }+ \\
\text { Propagación de la energia }\end{array}$ & $\begin{array}{l}41,2 \% \\
20,8 \%\end{array}$ & $\begin{array}{l}\text { Onda con que se propaga cl sonido } \\
\text { Si es muy potente, puede romper los objetos de } \\
\text { crist al } \\
\text { Energfa que va disminuyendo } \\
\text { en intensidad cuando se aleja del foco }\end{array}$ \\
\hline M4 $(97,5 \%)$ & $\begin{array}{l}\text { Propagación por aire } \\
\text { Detección }\end{array}$ & $\begin{array}{l}40,4 \% \\
23,8 \%\end{array}$ & $\begin{array}{l}\text { El sonido se propaga a través de las ondas } \\
\text { Las particulas chocan bruscamente con el cristal, } \\
\text { que vibra demasiado y se rompe }\end{array}$ \\
\hline
\end{tabular}

A estos resultados puede contribuir de un modo esencial la enseñanza formal y, en concreto, los tópicos de ondas y óptica.

- Propagación en medios materiales: cuando se requiere de los alumnos que expliquen la onda expansiva producida por un artefacto (ítem 5), se detecta una clara evolución, desde la imprecisión de responsabilizar de los efectos a un «ruido muy fuerte», en los alumnos más jóvenes, hasta las explicaciones más científicas: «energia que va disminuyendo en intensidad cuando se aleja del foco» o «las partículas chocan bruscamente con el cristal, que vibra demasiado y se rompe». No obstante, en todos los casos se hace jugar un papel preferente a la propagación por ondas, sin que éstas se concreten de un modo preciso como «las partículas del medio en igual estado de vibración» o "sucesivas compresiones y dilataciones del medio gaseosom.

En el caso de la propagación por medios sólidos, tierra y vía del tren (ítem 7), existe la creencia generalizada de que se produce una mejora en la propagación del sonido (ondas sonoras o vibraciones) frente a la que tiene lugar en el aire, lo que sólo los alumnos mayores traducen en una mayor velocidad de propagación. Persisten explicaciones meramente descriptivas («el sonido se propaga por la tierra o las vías") y sólo algunos alumnos de la muestra M3 prevén la posibilidad de conocer la proximidad de los agentes sonoros («si la onda está baja, están muy lejos; $y$, si es fuerte, más cerca»). Sin embargo, no se intuyen las explicaciones científicas, tales como $\mathrm{el}$ que los agentes sonoros generen ondas superficiales que se propagan con menor pérdida de intensidad que en el caso de la propagación a través de la atmósfera.

- Reflexión del sonido: el eco. El ítem 6, relativo a los lugares en que los alumnos han experimentado el eco y las condiciones para su producción, muestra una frecuencia muy elevada de respuestas que evidencian la vivencia personal de dicho fenómeno, lo que se traduce fundamentaimente en la identificación de lugares tales como montañas y espacios grandes y vacíos. Las condiciones que deben verificarse para el mismo sufren un ligero descenso en su frecuencia de respuesta y evolu- 
Tabla VI

Respuestas dadas por los alumnos al item 13 relativo al sonido producido por unas copas que contienen distinta cantidad de líquido (en el agrupamiento de las respuestas no se recogen aquéllas con porcentajes menores de un 10 por ciento).

\begin{tabular}{|c|c|c|c|c|}
\hline $\begin{array}{l}\text { MUESTRA } \\
\text { (Porcentaje de } \\
\text { respuestas) }\end{array}$ & $\begin{array}{l}\text { CATEGORÍA } \\
\text { DE LA } \\
\text { RESPUESTA }\end{array}$ & $\begin{array}{l}\text { PORCENTAJE } \\
\text { DE RESPUESTA }\end{array}$ & $\begin{array}{l}\text { AGRUPAMIENTO } \\
\text { DE LAS RESPUESTAS }\end{array}$ & $\begin{array}{l}\text { PORCENTAJE } \\
\text { DE RISPUESTAS }\end{array}$ \\
\hline MI $(100 \%)$ & $\begin{array}{l}\text { Sí } \\
\text { No }\end{array}$ & $\begin{array}{l}87,9 \% \\
12,1 \%\end{array}$ & $\begin{array}{l}\text { Cuanto más líquido, el sonido es más grave } \\
\text { Depende del líquido que contenga } \\
\text { Cuanto más líquido, el sonido es más agudo } \\
\text { Cuanto menos líquido, al tener más aire, } \\
\text { produce más sonido }\end{array}$ & $\begin{array}{l}28 \% \\
20 \% \\
16 \% \\
16 \%\end{array}$ \\
\hline $\operatorname{M} 2(97 \%)$ & $\begin{array}{l}\text { Si } \\
\text { No }\end{array}$ & $\begin{array}{r}93,7 \% \\
6,3 \%\end{array}$ & $\begin{array}{l}\text { Cuanto más líquido, el sonido es más grave } \\
\text { El líquido perturba la propagación del sonido }\end{array}$ & $\begin{array}{l}39,1 \% \\
34,8 \%\end{array}$ \\
\hline M3 $(96,2 \%)$ & $\begin{array}{l}\text { Sí } \\
\text { No }\end{array}$ & $\begin{array}{r}100 \% \\
0 \%\end{array}$ & $\begin{array}{l}\text { Cuanto más líquido, el sonido es menor } \\
\text { Cuanto más líquido, el sonido es más grave } \\
\text { El sonido varía debido a que el medio } \\
\text { también lo hace }\end{array}$ & $\begin{array}{l}32 \% \\
28 \% \\
24 \%\end{array}$ \\
\hline M4 (100\%) & $\begin{array}{l}\text { Si } \\
\text { No }\end{array}$ & $\begin{array}{r}100 \% \\
0 \%\end{array}$ & $\begin{array}{l}\text { Cuanto más lfquido, el sonido es más grave } \\
\text { y cuanto menor, más agudo } \\
\text { Cuanto más líquido, el sonido es más agudo } \\
\text { El sonido varfa debido a que el medio } \\
\text { también lo hace }\end{array}$ & $\begin{array}{l}43,2 \% \\
16,7 \% \\
16,7 \%\end{array}$ \\
\hline
\end{tabular}

cionan, desde una simple descripción del fenómeno ( «las ondas del sonido chocan con distintos obstáculos y éste se repite») hacia una analogía mecánica («el sonido rebota en el obstáculo») entre los alumnos mayores, surgiendo en este último caso argumentaciones más cientificas («el sonido no es absorbido»; «las ondas han de recorrer una distancia mínima hasta el obstáculo para que se produzca el eco»), fruto sin duda de las actuaciones docentes.

\section{Contaminación acústica}

El sonido como agente contaminante es tratado en el ítem 8 mediante la pregunta directa sobre si influye o no en la vida cotidiana de los alumnos. Los resultados muestran una respuesta mayoritariamente afirmativa, que destaca especialmente en los individuos de mayor edad. En cuanto a la particularización de los responsables de la contaminación, son señalados, en primer Ingar, toda clase de vehículos y, en menor medida, los gritos, Io que no varía sustancialmente con el nivel educativo de las muestras.

\section{Tecnología y aplicaciones acústicas}

Si comenzamos hablando de las aficiones musicales de los componentes de las distintas muestras (ítem 12), aquéllas sufren una notable disminución en variedad y cantidad a medida que crece su edad, excepción hecha de la muestra M4 cuyos miembros obligatoriamente han de saber interpretar con la flauta en su formación musical incluida en el currículo.

Colateralmente, las propiedades musicales de copas que contienen distinta cantidad de líquido (ítem 13) suelen ser agrupadas en torno al tono del sonido emitido (grave/agudo) dependiendo de la cantidad de líquido, lo cual evidencia un significativo error de interpretacín («cuanto más líquido, el sonido es más agudo») y está justificado de forma genérica por el hecho de que el medio varie en función de la cantidad de líquido que contiene. Parece evidente, pues, una escasa experiencia sensorial en el terreno de las propiedades acústicas de distintos emisores sonoros con una masa vibrante variable (por ejemplo, diapasón, varillas, xilófono, etc.). 
En cuanto a los elementos propios de un equipo o cadena musical (ítem 11), parecen ser bien conocidos el mando del volumen y el ecualizador/tono por los posecdores de tales aparatos, aunque no suelen percatarse del mando de balance. Por el contrario, los fundamentos básicos del micrófono y el altavoz (ítem 10) sólo empiezan a ser atisbados por los alumnos de más edad (micrófono: "transforma el sonido a través de una membrana en electricidad"), con lo cual se registra un alto grado de ausencia de respuestas en gran parte de los alumnos.

Finalmente, las aplicaciones lúdico-técnicas se centran en el fundamento del telćfono de hilo (ítem 14) y cl estetoscopio (ítem 15). En el primer caso, el papel del hilo como transmisor del sonido es reconocido por gran parte de los alumnos y, en menor grado, se alude a sus propiedades para justificar sus ventajas frente a la propagación por el aire; sólo algunos de los alumnos más jóvenes establecen la necesidad de tensar el hilo, sin duda por haber realizado dicha experiencia en un tiempo no muy remoto. Por lo que respecta al estetoscopio, éste es reconocido como receptor de «sonidos internos del cucrpo humano», especialmente procedentes del corazón, con un cierto poder amplificador atribuido por los alumnos mayores. La explicación de su funcionamiento es abordada por un escaso número de alumnos, que vuelven a reiterar su poder amplificador o responden de forma meramente descriptiva.

\section{IMPLICACIONES DIDÁCTICAS}

De los datos extraídos de la resolución del test y de su discusión cabe hacer algunas recomendaciones al profesorado que irían destinadas a suplir las carencias didácticas detectadas en este trabajo y que, resumidamente, podemos establecer a continuación:

1) En primer lugar debemos reivindicar un lugar más significativo de la acústica en los programas educativos, lo que fundamentamos en argumentos como los siguientes:

- Constituye un factor de desarrollo de las habilidades psicomotoras, tales como la audición, el lenguaje, la sensibilidad musical, etc., básicas para una normal evolución cognitiva y afectiva de los individuos.

- Es un núcleo interdisciplinar que integra materias tales como la física, Ia fisiología, Ia Iengua, la música, etc.

- Es vertebrador de actividades curriculares propias de la educación tecnológica o de Ciencia-TecnologíaSociedad (CTS), tal y como se ha puesto de manifiesto en las respliestas de algunos f́tems del test.

- Supone una ejemplificación idónea del movimiento ondulatorio que, junto al movimiento de partículas, constituye la base de la transferencia de energía en física.
- Como unidad temática cuenta con una estimable cantidad y variedad de trabajos prácticos descritos en la literatura educativa.

2) A continuación vamos a centrarnos en algunas recomendaciones de carácter más particular que deberían contribuir a fomentar un cambio conceptual significativo a la luz de algunas concepciones erróneas o superficiales descritas con antelación:

- Desde los primeros años de la escolarización obligatoria debe insistirse en actividades lingüísticas y sensoriales destinadas a la distinción ruido-sonido y a la descripción de este último a través de sus atributos (intensidad-tono-timbre). Sin duda a ello debería contribuir la incorporación de profesores especialistas en educación musical a los distintos centros de enseñanza.

- El reconocimiento del medio de propagación (aire, agua, hicrro, etc.) es esencial para identificar el sonido como un fenómeno físico con entidad propia. Asimismo debiera introducirse progresivamente el concepto de onda y movimiento ondulatorio mediante actividades prácticas (por ejemplo, ondas superficiales en agua) hasta su adquisición como modelización de una realidad (ondas mecánicas y electromagnéticas).

- Resultaría factible la medición de la velocidad de propagación del sonido y la interpretación del eco mediante experiencias en vivo.

- A los aiumnos de educación secundaria se les puede ir introduciendo en actividades de modelización del sonjdo a través de la teoría particular de la materia, que debería permitir, progresivamente, una explicación y predicción del fenómeno sonoro en sus diversas manifestaciones, tales como la velocidad de propagación en distintos medios materiales.

- El sonido generado en las ciudades se ha convertido en un agente contaminante de primera magnitud; su inclusión curricular puede permitir abordar de modo incipiente actividades de educación ambiental ( «ambientalización del currículo»), en una secuencia tal como lá siguiente: toma de conciencia del problema $\rightarrow$ estudio del mismo $\rightarrow$ acciones para contribuir a una mayor calidad del medio.

- Finalmente resulta deseable llevar al aula cl estudio de instrumentos lúdico-tecnológicos relacionados con el sonido (cadenas musicales, micrófonos, altavoces, teléfono de hilo, estetoscopio...) y aprender a interpretar su utilización a la luz de los conceptos y leyes de la acústica, de forma que el alumno conecte de un modo vivencial el auia con el medio social y cultural.

3) Podríamos finalizar haciendo referencia a algunas implicaciones curriculares de la acústica en el nivel educativo español en el que aparece con un cicrto peso específico; es decir, el nuevo bachillerato-LOGSE. Así, el RD \$179/1992, de 2 de octubre, donde se cstablece el currículo de aquél, en la asignatura de Física (contemplada en las modalidades de Ciencias de la Naturaleza y 


\section{Tabla VII}

Propuesta de contenidos de acústica que podrian ser utilizados para el desarrollo curricular del tema «Vibraciones y Ondas» incluido en el Real Decreto que establece el nuevo bachillerato.

\begin{tabular}{|c|c|}
\hline EPIGRAFES DEL. TEMA «VIBRACIONES Y ONDAS» & TÓPICOS DE ACÚSTICA \\
\hline $\begin{array}{l}\text { Movimiento vibratorio: el movimiento vibratorio } \\
\text { armónico simple }\end{array}$ & Emision del sonido (vibraciones de las partículas) \\
\hline $\begin{array}{l}\text { Movimiento ondulatorio } \\
\text { Magnitudes y características de las ondas }\end{array}$ & Propagación del sonido. Ondas sonoras. Magnitudes \\
\hline Ecuaciones de las ondas armónicas & Ecuación de las ondas sonoras \\
\hline $\begin{array}{l}\text { Fistudio cualitativo de algunas propiedades de las ondas: } \\
\text { reflexión, refracción, difraccion e interferencias. } \\
\text { Principio de Kuygens. Ondas estacionarias }\end{array}$ & $\begin{array}{l}\text { Propicdades de las ondas sonoras: } \\
\text { - Reflexion y eco } \\
\text { - Refraccion } \\
\text { - Interferencias y ondas estacionarias } \\
\text { - Difracción } \\
\text { - Principio de Huygens } \\
\text { - Efecto Doppler }\end{array}$ \\
\hline & Detección del sonido: acústica fisiológica \\
\hline Contaminación sonora, sus fuentes y efectos & $\begin{array}{l}\text { Aplicaciones del sonido: } \\
\text { - Contaminación acústica } \\
\text { - Aplicaciones médicas } \\
\text { - La física del sonido musical } \\
\text { - Acústica arquitectónica } \\
\text { - Ultrasonidos } \\
\text {... }\end{array}$ \\
\hline
\end{tabular}

de la Salud, y en la de Tecnología), aparece el tema 4, denominado «Vibraciones y Ondas», que incorpora los siguientes apartados:

- Movimiento ondulatorio: el movimiento vibratorio armónico simple.

- Movimiento ondulatorio. Magnitudes y características de las ondas. Algunas propiedades: reflexión y refracción. Ecuación de las ondas armónicas. Aplicaciones de las ondas en el mundo actual.

- Estudio cualitativo de algunas propicdades de las ondas: reflexión, refracción, difracción e interferencias. Principio de Huygens. Ondas estacionarias.

- Contaminación sonora, sus fuentes y efectos.

Por primera vez en los distintos niveles educativos no universitarios se intenta familiarizar a los alumnos con los fenómenos ondulatorios que, no lo olvidemos, cons- tituyen uno de los dos grandes mecanismos de transferencia energética en la naturaleza y que además permiten al ser humano obtener la mayor parte de la información sobre su entorno. A su vez no podemos confiar en que la abstracción del lenguaje físico-matemático inherente a la mecánica ondulatoria pueda generar un aprendizaje significativo sin contar con los apoyos sensoriales y cognitivos previos del alumno. Es aquí donde nuestra propuesta de actuación nos lleva a reclamar la incorporación de la acística como tópico modelo para articular la enseñanza del tema de "Vibraciones y Ondas», teniendo en cuenta además que el tema 5 contemplado en el Real Decreto aludido se dedica a la óptica, con lo que la acústica puede jugar un relevante papel como puente cognitivo hacia aquélla, habida cuenta que el carácter ondulatorio de la luz resulta más complejo de evidenciar (interferencias, difracción, etc.).

Vamos a desarrollar en la tabla VII los distintos contenidos acústicos que podrían servir de aplicación en el aula de los epígrafes propuestos por el Real Decreto citado, 
añadiendo aquellos tópicos precisos para proporcionar coherencia al tema de Vibraciones y Ondas, tomando para ello como referencia la secuencia sonora: emisión $\rightarrow$ propagación $\rightarrow$ detección.

\section{AGRADECIMIENTOS}

El autor desea expresar su gratitud a los profesores José Puerta y Antonio José Fernández por su colaboración en la aplicación del test.

\section{REFERENCIAS BIBLIOGRÁFICAS}

DRIVER, R. y WATTS, M. (1990). Rescarch on students' conceptions in science: a bibliography. School of Education: University of Leeds.

JUNCOS DEI, EGIDO, P. y PÉREZ DE LANDAZÁBAL, M.C. (1985). Un enfoque histórico y tecrológico para la enseñanza de la óptica en el BUP, en Perspectiva de la enseñanza de la óptica en España. Madrid: E. Bernabeu. SEDO.

LANZARICA, M.T., LOZANO, A., PARDO, R., PAREIO, C. y VII.CHES, J. (1978). Encuesta sobre el estado actual de la enseñanza de la física y la química en el BUP. Análisis y comentarios. Simposio sobre Didáctica de la Física y la Química. Madrid: INCIE.

LINDER, C.J. y ERICKSON, G. (1989). A study of tertiary physics students' conceptualizations of sound. International Joumal of Science Education, 11, pp. 491-501.

LINDER, C.J. (1992). Understanding sound: so what is the problem? Physics Education, 27, pp. 258-264.

IJINDER, C.J. (1993). University physics students' conceptualizations of factors affecting the speed of sound propagation. International Journal of Science Education, 15, pp. 655-662.
LORD, P. (1981). The challenge of Acoustic. Physics Education, 16, pp. $11-15$

LUCAS, A.M. (1988). Public knowledge of elementary physics. Physics Education, 23, pp. 10-16.

PERALES, F.J. (1987). Análisis de contenidos en óptica geométrica. Enseñanza de las Ciencias, 5, pp. 211-219.

PERALES, F.J. y NIEVAS, F. (1990). Un enfoque constructivista en la enseñanza de la óptica geométrica. Unjversidad de Granada: ICE.

PERALES, F.J. (1994). La enseñanza de la óptica, alambique. Didáctica de las Ciencias Experimentales, 1, pp. 133-137.

PFUNDT, H. y DUIT, R. (1994). Bibliography. Students' altemative frameworks and science education. Kiel: Institute for Science Education.

ROIG, J. (1982). El sonido, un tema olvidado. Cuadernos de Pedagogía, 90, pp. 54-56.

STEAD, B.V. y OSBORNE, R.J. (I980). Exploring students' concepts of Iight. Australian Science Teacher Joumul, 26, pp. $84-90$.

[Artículo recibióo en septiembre de 1995 y aceptado en abril de 1997.] 
ANEXO I

\section{LIBROS DE FÍSICA REVISADOS PARA EL ANÁLISIS DE CONTENIDOS DE ACÚSTICA}

CATALÀ, J., (1977). Física. Valencia: Saber.

FIDALGO, J.A., (1976). Física y Química $\left(2^{\circ} B U P\right)$. León: Everest.

GONÇALVES DE ALVARENGA, B. y MAXIMO, A., (1976). Física General. México: Harla.

SEARS, F.W. y ZEMANSKY, M.W., (1970). Fisica General. Madrid: Aguilar.

TIPLER, P.A., (1978). Física. Barcelona: Reverté.

VIDAL, J.M., (1972). Curso de Física. Barcelona: Grafesa.

\section{ANEXO II \\ LIBROS DE TRABAJOS PRÁCTICOS SOBRE ACÚSTICA REVISADOS}

AISBERG, E., (1969). La Fisica en la vida cotidiana. Barcelona: Danae.

BARR, G., (1971). Experiencias cientificas. Buenos Aires: Kapelusz.

BARR, G., (1971). Pruebas y juegos cientificos. Buenos Aires: Kapelusz.

CATHERALL, E.A. y HOLT, P.N., (1970). Experimentos con luz y sonido. Madrid: Santillana.

CHERRIER, F., (1977). Experimentos de física recreativa. Valencia: Mas-Ivars.

FESQUET, A.E.J., (1978). El sonido. El magnetismo. Buenos Aires: Kapelusz.

JÜRGEN, H., (1981). Experimentos con la ciencia. Madrid: Fuenteantigua.

MANDEL, M., (1979). Física recreativa. Madrid: Altea.

UNESCO, (1959). Manuel de L'Unesco pour l'Enseignement des Sciences. París: Unesco.

\section{ANEXO HII \\ PRUEBA DE CONOCIMIENTOS PREVIOS EN ACÚSTICA}

Centro

Cutso

Fecha

Apellidos

Nombre

Edad

Esta prueba va a servir para hacernos una idea de tus conocimientos sobre distintos aspectos del sonido a fin de mejorar la enseñanza de esta materia. Por favor, contesta reflexivamente a cada una de las siguientes preguntas. En caso de desconocer la respuesta, deja la cuestión en blanco. Si te falta espacio para alguna respuesta, sigue por detrás de la hoja. Gracias por tu colaboración.

1. El mundo en el que nos movemos no es un espacio siłencioso, sino lleno de múltiples sonidos. ¿ Sabrias decir en qué consiste el sonido? Explícalo con tus propias palabras.

2. ¿Qué palabras utilizas habitualmente para describir los sonidos y, por tanto, distinguir unos de otros? Escríbelas a continuación. 
3. Cuando se produce un sonido -por ejemplo, golpeando una campana-, éste se propaga por el aire a una cierta velocidad. Si conoces el valor aproximado de ésta, escríbelo a continuación.

4. Supón que eres un astronauta que ha llegado con un compañero a la Luna. Si le hablas, ¿crees que te escucharía igual que si estuviéseis en la Tierra? Comenta seguidamente tu respuesta.

5. Seguro que al ver u oir las noticias en radio o televisión puedes recordar una frase como la que sigue: En el día de ayer se produjo la explosión de un potente artefacto. La onda expansiva provocó la rotura de numerosos cristales en las viviendas próximas. ¿Qué crees que significa eso de la "onda expansiva»? Descríbelo con tus propias palabras.

6. Alguna vezen tu vida habrás experimentado el «eco». Describe a continuación el sitio en que ocurrió y por qué piensas que suceủe en unos lugares y en otros no.

Descripción del sitio

¿Por qué surge el eco en unos lugares y en otros no?

7. Supongo que habrás visto en alguna película un «indio» que pone su oreja sobre la tierra para saber si viene la caballería, o a algún cuatrero hacerlo en la vía del tren. ¿Por qué actúan así? Explica tu respuesta

8. Pienso en que estarás de acuerdo con que el ruido es un sonido, generalmente desagradable. ¿Normalmente tu vida se ve afectada por los ruidos?

Sî $\square$ No

¿Cuátes son los ruidos que más te molestan?

9. Cuando se producen tormentas con «gran aparato eléctrico» suelen observarse los rayos y oírse los truenos. ¿Por qué primero se ve el rayo y al cabo de un cierto tiempo se oye el trueno? Explícalo.

10. Si eres aficionado a escuchar música, habrás observado micrófonos y altavoces. Explica como crees que funcionan cada uno de estos instrumentos. 
Micrótono

Altavoz

11. Si dispones de un equipo de sonido en tu casa sabrás cómo se controla el sonido que emiten sus altavoces. ¿De qué mandos dispone y para qué sirven?

12. ¿Sabes tocar algún instrumento musical? En caso afirmativo escribe cuál o cuáles.

13. Se dispone de las copas que aparecen en la figura, iguales pero conteniendo distinta cantidad de un mismo líquido. Responde si crees que al golpearlas con un mismo objeto cambiará o no el sonido emitióo por ellas. En caso afirmativo describe esa variación.

$\square$ Sí $\square$ No
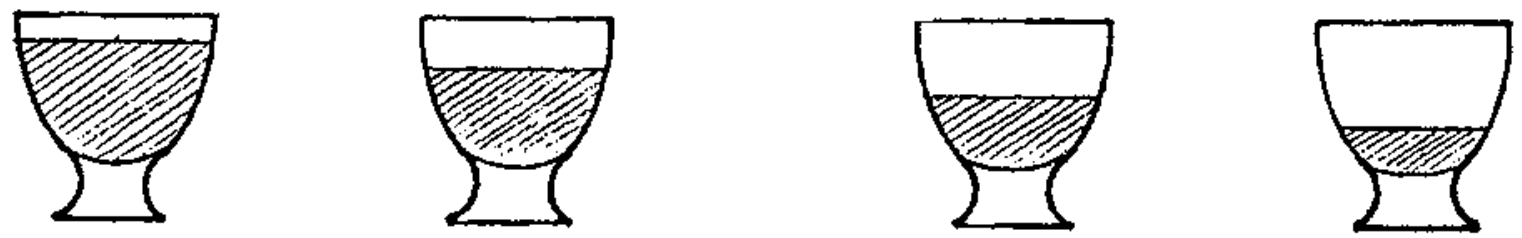

14. En alguna ocasión habrás construido con aigún amigo un «teléfono de hỉo» usando dos vasos de plástico y un trozo de hilo que une sus fondos (mira la figura). ¿Cómo crees que funciona? ¿Por qué? Explícalo.

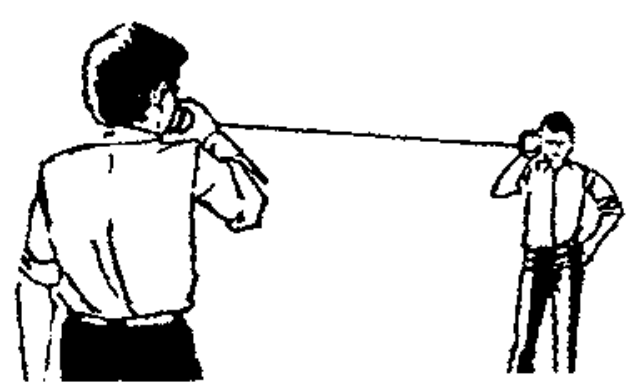

15. Cuando estás enfermo y acudes al médico, suele auscultarte con un instrumento que se llama estetoscopio (o fonendoscopio). ¿Para qué sirve? ¿Cómo crees que funciona? Explícalo. 


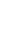

